\title{
La mondialisation foncière au Vietnam et au Cambodge. Une entrée par le droit dans la production de l'espace mondialisé
}

The globalisation of Land Law in Vietnam and Cambodia - exploring law making to understand the production of globalised space

Land Globalisierung im Vietnam und Kambodscha. Die Herstellung des globalisierten Raums im Licht des Rechtes

\section{Marie Mellac}

\section{OpenEdition}

Journals

Édition électronique

URL : http://journals.openedition.org/rge/8018

DOI : $10.4000 /$ rge.8018

ISSN : $2108-6478$

Éditeur

Association des géographes de l'Est

Référence électronique

Marie Mellac, « La mondialisation foncière au Vietnam et au Cambodge. Une entrée par le droit dans la production de l'espace mondialisé ", Revue Géographique de l'Est [En ligne], vol. 58 / 1-2 | 2018, mis en ligne le 25 avril 2019, consulté le 08 septembre 2020. URL : http://journals.openedition.org/rge/8018 ; DOI : https://doi.org/10.4000/rge.8018

Ce document a été généré automatiquement le 8 septembre 2020

Tous droits réservés 


\title{
La mondialisation foncière au Vietnam et au Cambodge. Une entrée par le droit dans la production de l'espace mondialisé
}

\author{
The globalisation of Land Law in Vietnam and Cambodia - exploring law making \\ to understand the production of globalised space \\ Land Globalisierung im Vietnam und Kambodscha. Die Herstellung des \\ globalisierten Raums im Licht des Rechtes
}

Marie Mellac

1 L'objectif de cet article est d'éclairer un moment spécifique de la production de l'espace mondialisé, celui de la fabrique des lois foncières. Il s'inscrit dans la filiation des travaux de Lefebvre (2000 [1974]) qui considère l'espace comme un produit social et un instrument du pouvoir, l'espace mondialisé étant conçu ici comme la manifestation spatiale (à la fois objectif et résultat) de la domination du modèle socio-économique capitaliste et libéral. Le choix qui est fait de donner de l'importance à ce moment spécifique se comprend de deux façons.

2 Le premier argument réside dans le contexte de la mondialisation foncière caractérisé par la convergence au niveau mondial des institutions foncières vers la propriété privée comme régime unique d'appropriation (Jones, 2003). Ce processus est porté par le très grand nombre d'Etats, surtout au Sud, qui ont entamé la révision de leurs législations foncières en faveur de la propriété privée au cours des dix à quinze dernières années (Le Roy, 2011). Loin des réformes distributives des années 1960 et 1970, les «contre-réformes» (Castellanet et Diepart, 2015) qui sont en cours, transforment les terres en biens marchands et les libèrent au bénéfice des investisseurs (Sassen, 2014) à un moment où ceux-ci s'intéressent particulièrement à elles et aux ressources agricoles (Chouquer, 2012). Ces réformes rendent possible les appropriations de terre à grande échelle et favorisent les processus de concentration et donc 
d'exclusion, provoquant de nombreux conflits, certains très virulents. Ces conflits sont d'autant plus actifs que le droit foncier a, en traitant de la propriété et de la terre, une dimension politique forte qui n'échappe plus aux ONG et les incite à rejoindre les organisations de développement bi- et multilatérales pour tenter d'influencer les Etats. Même réduite à l'écriture des textes, la fabrique du droit foncier constitue un moment particulièrement propice à l'observation des jeux d'acteurs qui encadrent la mondialisation foncière.

3 S'intéresser à la fabrique du droit positif permet aussi de prêter attention à sa dimension cognitive comprise comme "sa capacité à configurer les catégories avec lesquelles nous concevons la réalité » (Melé, 2009: 7). Si l'espace des textes n'est pas celui des sociétés qui est beaucoup plus complexe et ne se comprend pleinement qu'en dépassant la dichotomie artificielle entre ses dimensions physiques et abstraites et en tenant compte de la diversité de ses usages (Lefebvre, 1974, cité par Butler, 2009 : 145), il en participe à plusieurs titres : il le répercute à travers le travail des producteurs du droit qui le représentent dans le cadre d'un projet politique donné ; il agit sur les façons de le penser et de l'agir par sa présence même, son existence comme ressource, dans un processus de "légalisation de l'espace» (legalization of space) (Blomley, 2003). Projet pour et par l'espace, l'espace des textes est une fiction d'espace qui s'inspire de l'espace et tend à s'y actualiser dans un mouvement incessant de mise en ordre "géo-légale " (Forest, 2009). Étudier le contenu des textes et la façon dont ils ont été produits permet donc de révéler les représentations de l'espace et les jeux d'acteurs qui se construisent dans et autour de l'Etat pour produire cet espace. Quelle géographie s'écrit alors? Qui l'écrit et pour quel projet de pouvoir? S'agissant de lois foncières, ce travail est d'autant plus révélateur que le droit foncier, même réduit aux modalités d'appropriation de la terre, comme c'est le plus souvent le cas dans le droit positif, est fondamentalement spatial puisqu'il pose le fonds de terre (partie de l'étendue) comme support premier d'appropriation des ressources, ce qui n'est pas le cas de bien des sociétés prémodernes, et est un des fondements de l'espace abstrait tel qu'exposé par Lefebvre (2000 [1974]).

4 En s'intéressant à la fabrique des lois foncières et à leur contenu dans deux pays, le Vietnam et le Cambodge, en transition mais aux trajectoires politiques bien différentes, cet article ambitionne de montrer la diversité des processus de production de l'espace mondialisé et le rôle notable joué par le droit positif dans cette diversité. La comparaison du Vietnam et du Cambodge constitue un «instrument d'optique» (Appadurai, 1996) qui permet d'observer, sur une période de 25 ans, les transformations multiples des législations foncières pour les rendre compatible avec l'économie de marché. Par souci de concision, le texte donne peu d'éléments contextuels préalables et revient rapidement sur le processus de décollectivisation avant de proposer trois éclairages différents des transformations du droit. Le premier s'intéresse à «ce que disent les lois » et consiste en une analyse des projets pour l'espace que contiennent les textes fonciers produits par les deux États. Le regard se déplace ensuite vers la production du droit comme contexte éclairant ce contenu. Outre la littérature grise et scientifique décrivant cette production, les matériaux proviennent d'entretiens réalisés auprès des producteurs du droit et de divers spécialistes du foncier ${ }^{1}$. La dernière approche consiste en une tentative de repérage des effets les plus manifestes du droit foncier sur l'espace de sorte à pouvoir revenir, en conclusion, sur l'intérêt que revêt 
une entrée par le droit pour éclairer la diversité des cheminements vers l'espace mondialisé.

\section{Ce que disent les lois, cheminements dans le droit vers la propriété privée et le marché foncier}

5 De 1979 à 1989, le Cambodge est sous tutelle vietnamienne et les premiers éléments de réformes sont logiquement assez similaires dans les deux pays. Ils se produisent dès le début des années 1980 sous la pression populaire et visent d'abord à rétablir un lien individuel entre les foyers et les terres au sein des coopératives. Dans les deux cas, ces réformes sont installées par des directives et des décisions émanant du Parti et il ne s'agit alors pas tout à fait de droit positif ni spécifiquement de foncier. Un véritable droit foncier apparait au tournant des années 1990 lorsque les deux gouvernements s'emparent simultanément de ces deux «outils» pour mener leurs réformes économiques et sociales. Les choix politiques sont cependant très différents et se traduisent dans le droit foncier. Débute alors une période durant laquelle les deux législations évoluent dans la même direction, vers la propriété privée, mais de façon dissemblable, tant en ce qui concerne l'intensité de leur production que leur contenu.

\section{A Le droit comme laboratoire vers le marché au Vietnam}

6 Au Vietnam, la loi foncière de 1987 est une des toutes premières lois publiées. Mais il est encore un peu tôt et cette première loi, contredite dès 1988 par la directive 10, passe totalement inaperçue. En 1993, le pays se dote d'une seconde loi foncière qui dégage définitivement le foncier des coopératives (Pillot, 1995) en reconnaissant aux foyers des droits de transferts des terres importants complétant le droit d'usage individuel. Le pays publie ensuite avec régularité deux autres lois (2003 et 2013), et produit un très grand nombre d'amendements, de décrets d'application et de circulaires si bien qu'en 2013500 textes découlant de la loi de 2003 étaient dénombrés (Phan 2014).

7 L'analyse diachronique du contenu des quatre lois montre que le droit est réformé par la marge : plusieurs principes fondamentaux sont maintenus d'un texte à l'autre et seules des dispositions apparemment annexes sont modifiées. Avec le temps, ces modifications se sont toutefois accumulées au point de modifier la valeur réelle des principes et de transformer fondamentalement le régime de propriété. Les textes sont de plus en plus longs (figure 1), de plus en plus techniques mais aussi de plus en plus complexes et contradictoires, les principes affirmés étant contredits par certaines dispositions et des exceptions. Mais ils ont permis un passage progressif vers l'économie de marché, compatible avec l'idéologie socialiste. 
Figure 1 - Chronologie simplifiée des textes fonciers du Vietnam et du Cambodge

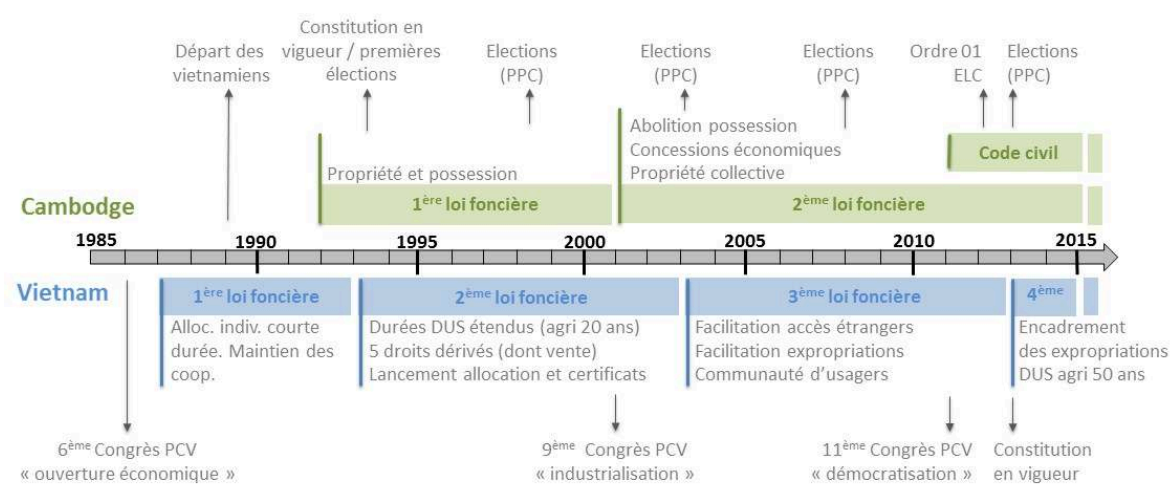

(réalisation M. Mellac, 2019)

8 Parmi ces principes, le premier est celui qui fait de la terre la "propriété du peuple entier, gérée par l'État » et en attribue en quelque sorte la propriété éminente, à forte valeur symbolique mais avec peu d'utilité, à l'État. De ce principe découle celui de l'allocation de droits d'usage des sols (DUS) sécurisés par des certificats temporaires. Dans un premier temps (1987), les droits sont réduits à l'usage (obligatoire) pour des durées encore imprécises mais courtes. Dans les lois suivantes, les durées s'allongent et les superficies maximales autorisées par individu augmentent (figure 2). Par ailleurs, le droit d'usage est enrichi de droits associés toujours plus nombreux de sorte qu'il ressemble de plus en plus à une propriété foncière qui aurait été constituée par ajouts successifs de droits. En conséquence, un marché foncier des DUS agricoles peut se développer dans un contexte de sécurité foncière, celle-ci ayant été aussi garantie par un vaste programme d'attribution de certificats ("carnets rouges») aux particuliers, lancé au milieu des années 1990 et vite réalisé sur les terres agricoles comme forestières (Mellac et al., 2010). 
Figure 2 - Catégories de terres, catégories d'usagers et cadres des droits d'usage individuel des terres agricoles dans les quatre dernières lois foncières du Vietnam

\begin{tabular}{|c|c|c|c|c|c|}
\hline \begin{tabular}{|l|} 
date \\
d'approbation
\end{tabular} & \begin{tabular}{|l|} 
nbre \\
d'article \\
s
\end{tabular} & catégories de terre & catégories d'usagers & $\begin{array}{l}\text { durée des DUI des terres } \\
\text { agricoles* (si limitée) }^{*}\end{array}$ & $\begin{array}{l}\text { superficie des DUI } \\
\text { terres agricoles* }{ }^{*} \text { s }\end{array}$ \\
\hline 1987 & 57 & $\begin{array}{l}5 \text { : terres agricoles ( } 2 \text { sous- } \\
\text { catégories), terres forestiè res, } \\
\text { terres d'habitation, terres à } \\
\text { usage spécifique et terres non } \\
\text { utilisées. }\end{array}$ & $\begin{array}{l}\text { 7: fermes d'état; entreprises } \\
\text { d'état; institutions; unités de } \\
\text { l'armée; coopératives; } \\
\text { groupes de production; } \\
\text { organisations sociales et } \\
\text { individur. }\end{array}$ & $\begin{array}{l}\text { non spécifiée mais } \\
\text { temporaire }\end{array}$ & $\begin{array}{l}\text { selon les disponibilté } \\
\text { foncières et le nomb } \\
\text { foyers des commun }\end{array}$ \\
\hline 1993 & 89 & $\begin{array}{l}\text { 6: terres agricoles; terres } \\
\text { forestières; terres } \\
\text { résidentielles rurales; terres } \\
\text { urbaines; terres à usage } \\
\text { spécifique; terres non } \\
\text { utilisées }\end{array}$ & $\begin{array}{l}3 \text { : organisations d'état } \\
\text { (organisations économiques, } \\
\text { sociales et politiques, unités } \\
\text { de larmée et institutions); } \\
\text { foyers; individus. }\end{array}$ & $\begin{array}{l}\text { cultures annuelles }=20 \\
\text { ans cultures pérennes }= \\
50 \text { ans }\end{array}$ & $\begin{array}{l}\text { (selon décret } 64 \text { de } \\
\text { cultures annuelles = } \\
\text { nord et } 3 \text { ha au sud; } \\
\text { cultures pérennes = } \\
\text { dans les plaines et } 3 \\
\text { les montagnes et co }\end{array}$ \\
\hline 2003 & 146 & $\begin{array}{l}3 \text { : terres agricoles ( } 8 \text { sous- } \\
\text { catégories dont } 3 \text { fore stières); } \\
\text { terres à usage non agricole; } \\
\text { terres dont fusage n'a pas } \\
\text { encore été déterminé }\end{array}$ & \begin{tabular}{|l|}
7 : organisations domestiques; \\
foyers et individus \\
vietnamiens; communautés; \\
institutions religieuses; \\
organisations étrangères \\
exerçant une fonction \\
diplomatique; vietnamiens \\
ayant une résidence \\
premanente à l'étranger; \\
personnes physiques et \\
groupements étrangers.
\end{tabular} & $\begin{array}{l}\text { cultures annuelles }=20 \\
\text { ans cultures pérennes }= \\
50 \text { ans; renouvelable sur } \\
\text { demande }\end{array}$ & $\begin{array}{l}\text { cultures annuelles = } \\
\text { cultures pérennes = } \\
\text { dans les plaines, 301 } \\
\text { les montagnes }\end{array}$ \\
\hline 2013 & 212 & 3: idem & $\begin{array}{l}7 \text { : organisations domestiques; } \\
\text { foyers et individus } \\
\text { vietnamiens; communautés; } \\
\text { institutions religieuses; } \\
\text { organisations étrangères } \\
\text { (gouvernementales et } \\
\text { intergouvernementales); } \\
\text { vietmaniens de l'étranger; } \\
\text { entreprises étr angères ou à }\end{array}$ & $\begin{array}{l}\text { cultures }=50 \text { ans } \\
\text { renouvelable sur demande }\end{array}$ & idem \\
\hline
\end{tabular}

* concerne les droits d'usage individuel (DUI) des terres agricoles attribué es aux foyers à titre gratuit (sans loyer). Les durées et superficie forestières et dans le cas de terres louées (possible à partir de 2003)

(réalisation M. Mellac, 2019)

Un autre principe fondamental de la législation foncière est celui de classification des terres et des usagers. Formant une sorte de matrice, le croisement des classes détermine les modes (allocation, location, achat, etc.) et conditions (superficie, durée, taxation, etc.) d'accès des différents types d'usagers aux différents types de terres. Présentes dès la loi de 1987, les classifications se retrouvent, partiellement modifiées, dans chaque nouveau texte et semblent évoluer comme si le législateur (ré)inventait progressivement la complexité foncière. Les catégories de terre sont fixées a priori dans le droit et c'est l'exercice de planification qui classe les terres, les catégories retenues pouvant, dans certains cas, être contraire à l'usage observé, de façon à orienter la mise en valeur. Les changements de catégories sont difficiles, en particulier pour les terres rizicoles qui sont protégées, et restent très encadrées mais ils sont graduellement assouplis. Au cours du temps, les catégories d'usagers sont quant à elles de plus en plus nombreuses et les conditions d'accès moins restrictives. Les quotas surfaciques qui permettaient d'éviter les processus de concentration des terres agricoles et forestières subsistent mais perdent peu à peu ce rôle puisque les particuliers peuvent accéder au foncier selon un nombre toujours plus grand de procédés (attribution gratuite ou onéreuse, achats, locations courtes ou longues, etc.) et peuvent ainsi constituer un " patrimoine » diversifié. En revanche, les expropriations d'usagers à des fins d'utilité publique sont progressivement facilitées, particulièrement en 2003, ce qui permet aux collectivités centrales et locales (provinces, villes, districts) de transformer la terre en capital pour financer le développement. Hautement contestées après 2003, les expropriations sont mieux encadrées dans le texte de 2013, mais restent néanmoins possibles dans un large éventail de situations, dont le développement économique. 
Avec le temps, les nouvelles dispositions portent sérieusement atteinte au caractère protecteur de la loi envers les petits exploitants et remettent en cause, sans toutefois l'annuler totalement, le principe de répartition égalitaire. Plus généralement, la législation desserre graduellement les liens - sans les supprimer - entre l'Etat et le foncier et rend possible la libéralisation progressive du marché foncier.

\section{B La propriété comme crédo au Cambodge}

Rien d'aussi complexe dans le Royaume du Cambodge qui maintient lui aussi le principe d'une propriété éminente de la terre revenant à l'Etat mais qui, comme beaucoup d'autres pays anciennement socialistes, n'en respecte pas le sens profond et installe rapidement un régime de propriété. Entre 1985 et 2010, Hap Phalthy (2010) recense un nombre important de textes ayant une incidence sur le foncier (186) mais les textes spécifiques sont moins nombreux qu'au Vietnam - deux lois foncières (1992 et 2001) et un code civil (2007) - et leur contenu n'a pas la même continuité. En 1992, au moment des accords de Paris, la première loi foncière définit plusieurs types de biens fonciers (estate) sur lesquels s'appliquent deux principaux types d'appropriation: propriété et possession. La possession, dont les juristes (Hel, 2012 et 2008 ; Hap, 2010) identifient la logique dans le Cambodge féodal précolonial avant d'être réinterprétée par le code civil de 1920, est un régime fort, aux caractéristiques similaires à la propriété pour les transferts mais contingent à l'usage continu des terres. Jusqu'alors permanent, ce régime devient transitoire avec la loi de 1992 qui lui donne vocation à se transformer en propriété après cinq années d'existence. Mais la loi limite encore la propriété au foncier bâti et crée de la confusion pour les terres agricoles dont le régime ne peut toujours pas évoluer. En 1993, une nouvelle (et dernière) Constitution reconnait la propriété pour toutes les terres et donc pour les terres agricoles. C'est alors le corpus juridique qui devient confus puisque la législation foncière reste non modifiée jusqu'à 2001, date de la seconde loi foncière. Celle-ci reconnait la propriété des terres agricoles et abolit en quelque sorte la possession (Hel, 2012) en interdisant toute nouvelle création à partir de sa promulgation. Les foyers ne peuvent donc plus constituer ou agrandir leur patrimoine foncier agricole (grâce à la création, par la reconnaissance de l'usage, de la possession), et ceux, très nombreux, qui possèdent encore des certificats de possession sont mis en insécurité par une propriété qui a plus de force.

11 La loi de 2001 révise par ailleurs les modalités de constitution des domaines privé et public. En 1992, la propriété publique apparaît sous la forme de listes énumérant les objets géographiques (forêts, pêcheries, monastères, etc.) ne pouvant pas relever de la propriété privée. Cette ébauche de formation d'un domaine public par énumération rappelle les classifications du Vietnam et peut être considérée comme une survivance de la période socialiste qui disparaît avec la loi de 2001 lorsque l'Etat devient propriétaire de «tous les biens [...] qui n'ont pas fait l'objet d'une appropriation privée régulière ou qui ne sont pas en cours d'appropriation privée par le biais d'une possession (...)» (art.12). A partir de cette date, la possession ne pouvant plus être reconnue, la propriété ne peut croître que par volonté de l'Etat en empiètement de son domaine privé. Il dispose pour cela de trois outils, la vente de ce domaine rendue possible par décret du Premier ministre, l'octroi de titres de propriété collective aux communautés indigènes et l'octroi gracieux de concessions ayant une utilité sociale (habitation / culture de subsistance), celles-ci pouvant ensuite être transformées en droits de propriété. Hel (2008) présente les deux dernières dispositions comme des 
compensations à l'abolition de la possession. Mais le Cambodge qui est très lent à attribuer des titres de propriété, l'est aussi considérablement pour créer ces deux types de biens ${ }^{2}$. Et le nouveau régime est moins dynamique que ne l'était la possession puisque les concessions ne peuvent, en aucun cas, découler d'une occupation de fait.

Fragilisés par les nouvelles dispositions, les foyers se retrouvent d'autant plus en insécurité que l'Etat se dote alors d'un second type de concessions, à caractère économique, qui lui permet de mettre à disposition du foncier - agricole surtout - de son domaine privé pour des projets d'exploitation de grandes dimensions. Ces concessions (Economic Land Concession ou ELC), attribuées en échange de loyers et accessibles aux étrangers, sont relativement contraintes: elles sont temporaires, révocables et doivent être exploitées de façon continue. Leurs durées d'attribution sont cependant très longues ( 99 ans devenus 50 ans en 2016) et les superficies autorisées très importantes (10 000 hectares avec dérogation possible). Elles produisent par ailleurs les mêmes effets que la propriété pendant leur existence et, si elles sont inaliénables, peuvent être transférées à la descendance en cas de décès. Alors que l'occupation n'a plus de force, le régime des concessions économiques proposé est l'un des plus favorables au monde. Contrairement au Vietnam où un tel régime concessionnaire n'existe pas et où les entreprises d'Etat (de plus en plus mixtes il est vrai) disposent seules de superficies agricoles importantes, la législation cambodgienne a grand ouvert le foncier agricole aux investisseurs privés. La description du dispositif juridique foncier cambodgien serait incomplète sans mentionner le code civil adopté en 2007 et entré en vigueur en 2011. Ce texte dont le contenu entre fréquemment en conflit avec le reste du corpus juridique inspiré par la common law (Kork, 2012), était encore largement méconnu des praticiens en 2016 et ne faisait pas référence. Représentant une réforme profonde, mais encore « de papier » pour des raisons expliquées plus bas, il ne sera pas décrit ici.

\section{Autour des textes : tensions et débats dans la fabrique du droit}

Pour comprendre certains des choix fonciers faits dans les deux pays et la façon dont les textes sont appliqués ou non, il faut en effet considérer le contexte d'écriture des textes et les tensions qui l'entourent, celles-ci se manifestant dans mais aussi " entre » les textes, par des continuités ou au contraire des sauts ou des incohérences. Dans les deux pays, trois temps peuvent être distingués : un premier temps d'écriture au plus haut niveau assez mal connu et expérimental avant 2000, un temps de montée en puissance des influences externes jusqu'à 2012 environ, un dernier temps de réaffirmation du pouvoir (exécutif) sur le droit et à travers le droit.

\section{A Les premiers temps exploratoires des législations foncières}

14 La production légale foncière du Cambodge des années 1980 et 1990 est mal documentée. Muets sur les premières directives, les experts restent discordants sur le cadre précis d'écriture de la loi foncière de 1992. Cette loi qui se caractérise par des ressemblances importantes avec le code civil colonial de 1920 (Hap, 2010b) et s'inscrit résolument dans la tradition du droit romano-germanique, bénéficie d'influences indirectes françaises et a engagé sans aucun doute une expertise juridique occidentale ${ }^{3}$. 
Elle s'est néanmoins faite sous contrainte politique forte ce qui se traduit par des hésitations encore fortes quant au régime d'appropriation à privilégier.

Alors que le Cambodge rejoint le monde libéral dans un contexte où les juristes nationaux sont absents (Kong 2012) mais les experts étrangers nombreux, le Vietnam des premiers temps ferme quant à lui volontairement le domaine foncier aux étrangers. $\mathrm{Au}$ milieu des années 1990, la Banque mondiale s'approche plusieurs fois de l'administration foncière lui proposant de délivrer des prêts à faibles taux afin de financer la modernisation du système de gestion foncière mais les offres sont rejetées par le gouvernement qui préfère utiliser le budget national (Dang Hung Vo et Palmkvisk, 2001). Alors que la coopération légale s'intensifie et que l'expertise juridique étrangère devient courante (Mellac et al., 2010), la coopération foncière se développe peu avec un seul programme suédois recensé, sans composante juridique et focalisé sur les aspects techniques (le SEMLA ou Strengthening Environmental Management and Land Administration). Les textes sont alors produits au plus haut niveau, au sein de la cellule juridique de l'Assemblée Nationale, et le processus de discussion reste relativement opaque. Pour les deux premières lois (1987 et 1993), le professeur Dang Hung $\mathrm{Vo}^{4}$, en charge de l'administration foncière de 2002 à 2007, indique un processus d'écriture très rapide. Un juriste et universitaire (Hanoi, novembre 2013), estime quant à lui que ni les membres de l'Assemblée ni le Premier ministre ne savaient vers quoi ils allaient, ni comment procéder, en lançant le processus d'écriture des deux textes. Le même affirme que l'insatisfaction des législateurs face aux textes est un des éléments les ayant incités à renforcer par la suite les coopérations (dont la formation) en matière juridique. Il précise aussi, ce que corroborent plusieurs entretiens, que les textes furent écrits et mis en application selon une logique expérimentale (« lois laboratoires»), ce qui explique à la fois le caractère peu abouti des textes initiaux et la multitude des décrets d'application et textes correctifs permettant de tester, sur des durées et dans des espaces choisis, certains éléments de nouveauté proposés. Chaque nouvelle loi intègre ensuite ce qui a fonctionné et poursuit l'expérimentation.

\section{B La montée des influences étrangères}

16 L'écriture des textes change à partir du travail sur la loi de 2003. Dang Hung Vo assure le secrétariat général d'une commission de préparation du projet de loi foncière (Drafting Land Law 2003) qui intègre pour la première fois un groupe d'experts occidentaux, dont des allemands et des suédois ${ }^{5}$. Selon ces experts, rencontrés en entretien en 2008, les allers et retours du texte pour commentaires produisent des effets sur son contenu mais les délais sont trop serrés et les marges de manœuvres étroites. Les commentaires ne permettent alors que de déplacer légèrement le curseur dans une direction ou dans une autre, sans revenir sur les choix fondamentaux faits au sommet (Mellac et al., 2010). Des débats réellement contradictoires et impliquant davantage d'experts étrangers n'apparaissent finalement que dix ans plus tard autour de la quatrième loi foncière.

$\mathrm{Au}$ Cambodge, l'importance des influences étrangères dans la rédaction de la loi de 2001 est attestée par tous les experts. Sa paternité reste néanmoins confuse, voire disputée, les experts, eux-mêmes de nationalités différentes, mentionnant l'influence déterminante d'une ou plusieurs coopérations ${ }^{6}$. Ces discordances reflètent des tensions entre coopérations étrangères qui se construisent tant à propos du foncier que du droit 
lui-même. Trzcinski et Upham (2012) mentionnent, pour le droit, l'existence de « waves of foreign experts » agissant au sein de différentes sphères d'influence, notamment celle, très libérale, de la Banque mondiale, et se référant à des façons de penser le droit pouvant différer de l'une à l'autre. Cela crée des tensions entre les différents « foreign patrons » agissant au Cambodge et entre les ministres ayant misé sur l'un ou l'autre de ces parrains (ibid.). La confusion des avis sur la loi de 2001 reflète aussi la complexité du processus de rédaction dont Hap (2010) mentionne qu'il a été très long : il a débuté en Français en impliquant des juristes français et cambodgiens formés en France et s'est poursuivi en plusieurs langues et en mobilisant apparemment plusieurs cercles de juristes après la publication d'un rapport critique produit par l'Asian Development Bank. Le texte est finalement issu d'un processus complexe de négociations entre les principaux bailleurs, qui prennent alors conscience de son caractère crucial, ainsi qu'entre les institutions cambodgiennes (Carrier, 2007), même s'il demeure une incertitude quant au niveau de participation de ces dernières (Williams, 2013). Le texte qui en découle est composite et inachevé et nécessite ensuite une quinzaine de décrets d'application (ibid.).

Alors que le Vietnam a attribué seul et rapidement des certificats fonciers dès le début des années 1990, le Cambodge qui choisit pourtant la propriété privée et bénéficie de financements étrangers très conséquents, accumule les retards de titrement que ce soit pour les individus ou les communautés indigènes. Entre 2001 et 2015, les retards sont tellement criants et la situation foncière tellement dégradée que la Banque Mondiale puis la coopération allemande, partenaires majeurs du ministère en charge du foncier (le « Ministry of Land Management, Urban Planning and Construction »- MLMUPC) ${ }^{7}$, sont attaquées successivement par des médias et des ONG et lui retirent momentanément leur soutien. De son côté, le Ministère de la Justice qui a délégué la production des deux codes pénaux aux Français, choisit d'élaborer un code civil et d'en confier la rédaction à la coopération japonaise (Kiyohara, 2012) afin d'équilibrer ses partenariats ${ }^{8}$. Cet arbitrage, sans lien avec le droit, introduit une nouvelle influence étrangère en matière foncière et aboutit à la production d'un texte d'une complexité totalement nouvelle pour le système légal cambodgien (Menzel, 2012) et porteur de conflits entre traditions juridiques (Kork, 2012). Il attise aussi les tensions déjà existantes entre les différents acteurs du foncier. Ainsi certains membres du MLMUPC, ministère créé peu de temps avant la loi de 2001 pour le mettre en application, feignaient encore, en 2016, d'ignorer le code civil.

\section{Passer par le droit pour régler les conflits}

19 Alors que les ministères et les coopérations jouent avec le droit, profitant pour cela du peu d'intérêt manifesté par le Premier Ministre Hun Sen pour son contenu, le Cambodge entre, à la fin des années 1990, dans une période de conflits fonciers intenses liés à la multiplication des dépossessions urbaines et rurales. Avec le temps, l'accumulation des violences et la multiplication des résistances tant internes qu'externes (médias en anglais, ONG nationales et étrangères, acteurs transnationaux, etc.), ces conflits deviennent un enjeu social et politique majeur (Diepart et Mellac, 2017) qui contraint Hun Sen à intervenir à plusieurs reprises afin de manifester son efficacité et sa bonne volonté. Ses interventions se concentrent quasi exclusivement lors des périodes pré-électorales et consistent à prendre des décisions attendues par la population mais dont les effets sont mal maîtrisés et peu vérifiables. En 2012, par 
exemple, le gouvernement a publié un ordre (dit ordre 01) annonçant un moratoire des concessions économiques assorti d'un processus de révision et suivi du lancement d'une campagne de titrement en faveur des foyers touchés par leur déploiement. Plus récemment, le MLMUPC a lancé une importante initiative de règlement des conflits fonciers largement relayée dans la presse. Mais les effets de ces décisions agissent parfois à l'encontre de ce qui était annoncé (Bourdier, 2016) et sont de courtes durées. "Ainsi, la chute du nombre des conflits fonciers enregistrée en 2013 par la Licadho fut immédiatement suivie, l'année suivante, par une hausse spectaculaire de la conflictualité provoquée par le non-respect des annulations, des processus de titrement trop hâtifs ainsi que par l'absence de titrement effectif dans les zones les plus conflictuelles " (Mellac et Diepart, 2017 : 138). Quant aux conflits, si certains ont trouvé une issue, ils continuent de faire la une de ce qu'il reste de la presse en anglais après les dernières élections (en 2018). Le pouvoir central mobilise donc le droit comme discours, pour créer un effet d'annonce pouvant lui être favorable, et entre à son tour dans un jeu qui augmente encore la confusion juridique et éloigne les textes de leur application.

Au Vietnam où le droit n'a pas créé de système concessionnaire, la production de la loi foncière de 2013 s'effectue dans un climat foncier beaucoup plus tendu lui aussi mais néanmoins plus ouvert qu'en 2003. La colère populaire s'est amplifiée avec l'augmentation rapide des expropriations (pour raison d'intérêt public) autorisées par la loi de 2003 et encouragées par le gouvernement pour financer le développement (Mellac et al., 2010). De son côté, la crise alimentaire mondiale de 2007-2008 a ouvert un débat interne sur l'autosuffisance alimentaire et sur la protection des terres agricoles, particulièrement des rizières. Début 2008, les questions foncières sont sur toutes les lèvres, les conflits majeurs sont médiatisés, la corruption est reconnue comme patente. La signature, en 2008 aussi, du premier projet de la Banque Mondiale concernant directement le foncier, indique un changement par rapport à la politique de fermeture antérieure. En 2013, la loi foncière et la Constitution arrivent sur le bureau de l'Assemblée nationale en même temps et occasionnent l'organisation de consultations populaires. La loi foncière recueille plus de 6 millions de réactions, la plupart à propos d'expropriations abusives (land grabs) de la part des autorités provinciales (ibid.). Le nouveau climat légal rend aussi possible un travail de consultations et de commentaires en dehors des dispositifs prévus par l'Etat. En octobre 2012, l'« Advocacy Coalition Support Programme », financé par la coopération anglaise et mené par Oxfam, organise des consultations à la base dans cinq provinces (Wells-Dang, 2013) et présente un plaidoyer en s'entourant de différents partenaires nationaux (ONG, agences publiques, organisations de masse, médias et experts dont Dang Hung Vo).

21 En juin 2013, la première version de la loi est rejetée par plus de $80 \%$ des députés (Thu, 2013). C'est la première fois qu'un texte n'est pas adopté lors de son premier passage devant l'Assemblée nationale. Cet échec est officiellement justifié par l'incompatibilité du texte avec la Constitution (ibid.) dont un nouvel amendement doit être approuvé à la fin de l'année. Mais peut-on vraiment croire à un tel manque d'anticipation de la part du législatif ? Des représentants d'Oxfam, (Wells-Dang et al., 2015) estiment que la force du plaidoyer a fait reculer les députés. Ce recul est préparé depuis quelques années déjà par la publication de rapports d'expertise, notamment de la Banque mondiale, pointant la corruption et les distorsions du marché liées aux interventions de l'Etat (Mellac et al., 2010). Des entretiens conduits en 2013 montrent aussi que certains députés étaient inquiets de la force des conflits et que des désaccords profonds existaient entre eux, 
notamment autour de la notion d'« intérêt public ». Lorsque les débats reprennent, le partenariat initié par Oxfam se transforme en une coalition ("land policy coalition » ou LANDA) qui anime une campagne particulièrement intense (Wells-Dang et al., 2015) à laquelle participent d'autres organisations internationales telles ActionAid ou la Banque mondiale qui emmènent à leur tour une partie des ONG locales.

Le texte de 2013 est finalement décevant pour les ONG (Wells-Dang et al., 2015). La loi adoptée intègre peu des modifications attendues et apporte peu de changements, conservant notamment la clause d'expropriation à fins d'intérêt économique. En dépit des pressions, l'Assemblée et, au-dessus d'elle le Parti, approuvent des textes s'inscrivant dans la continuité de leurs choix initiaux. Malgré la mise en visibilité des contre-pouvoirs internes et externes, il n'y a pas, encore aujourd'hui, de véritable autonomisation du droit foncier par rapport au Parti, et il n'existe toujours pas de propriété privée.

\section{Les effets du droit sur l'espace}

Que nous disent les textes sur l'espace alors que le droit, au Cambodge, est appliqué de façon problématique et qu'il est, au Vietnam, un instrument encore peu négociable du pouvoir? Dans les deux cas, il est frappant de constater que l'espace produit porte les traces du contenu du droit et de la façon dont le droit est travaillé à l'articulation entre pouvoir et société. Ces traces sont en partie communes et signalent, dans ce cas, les propriétés associées par Lefebvre (2000 [1974]) à l'espace abstrait (fragmentation, hiérarchie, géométrique, homogénéité ...) tel que produit par la modernité et le triptyque «Etat, droit et propriété privée ». Les espaces produits dans les deux pays ne sont cependant pas identiques et portent aussi les traces des façons différentes qu'ont les Etats de concevoir leur rôle dans le foncier à travers le droit.

\section{A Vers l'espace abstrait}

Sans surprise, les propriétés de l'espace abstrait qui se manifestent le plus visiblement dans les dynamiques spatiales observées au Cambodge et au Vietnam relèvent de la fragmentation et du géométrique, tous deux produits par l'urbanisation mais aussi par les allocations ou appropriations individuelles de terres en lien avec les titres ou certificats et relevés dans les cadastres. Dans les deux pays, il est notable aussi que le visuel supplante peu à peu le corps et la pratique dans la production de l'espace avec, notamment, la multiplication des murs délimitant les propriétés et celle des panneaux indiquant leur propriétaire ou leur état («à vendre » ou « à louer »). 


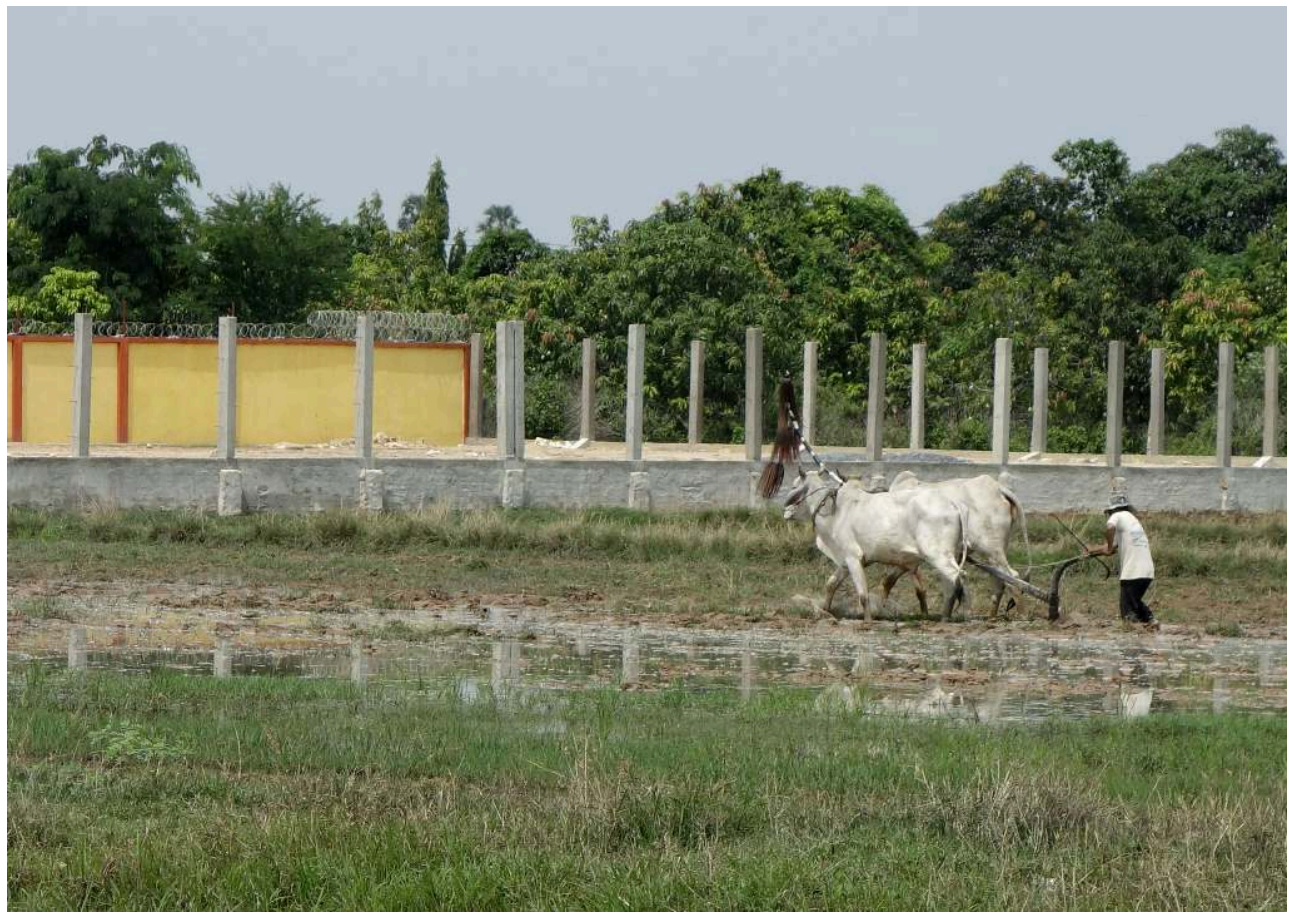

(cliché M. Mellac, 2015)

Dans la photographie ci-dessus (figue 3), un agriculteur des environs de Phnom Penh travaille la terre à proximité d'une parcelle enclose et remblayée qui entrave la circulation des hommes, des animaux et de l'eau. Ce type de parcelle, fréquent le long des routes, peut rester inutilisé durant des années, son nouveau possesseur se contentant de la fermer et de la surélever pour marquer sa propriété et être certain qu'elle ne sera plus mise en culture. Dans les rizières voisines, nul besoin d'un tel marquage et chacun se déplace librement, les ayants-droits étant connus de tous et l'état de la mise en culture indiquant qui des hommes, des bêtes et de l'eau, peut circuler ou non. Une parcelle isolée au milieu des champs est bien plus fréquente au Cambodge qu'au Vietnam où l'habitat ne peut pas progresser isolément en raison du classement des terres associé à la planification. Le passage du coutumier à la propriété y provoque néanmoins aussi le développement du marquage et un processus de parcellisation dans les espaces, notamment forestiers, qui n'étaient pas appropriés individuellement. Cela est particulièrement visible par exemple dans l'avancée du front pionnier agricole sur les hauts plateaux centraux à la frontière avec le Cambodge, marquant par là le contrôle de l'Etat (figure 5). 
Figure 4 - Les condominium « Ciputra » à Hanoi et Phnom Penh

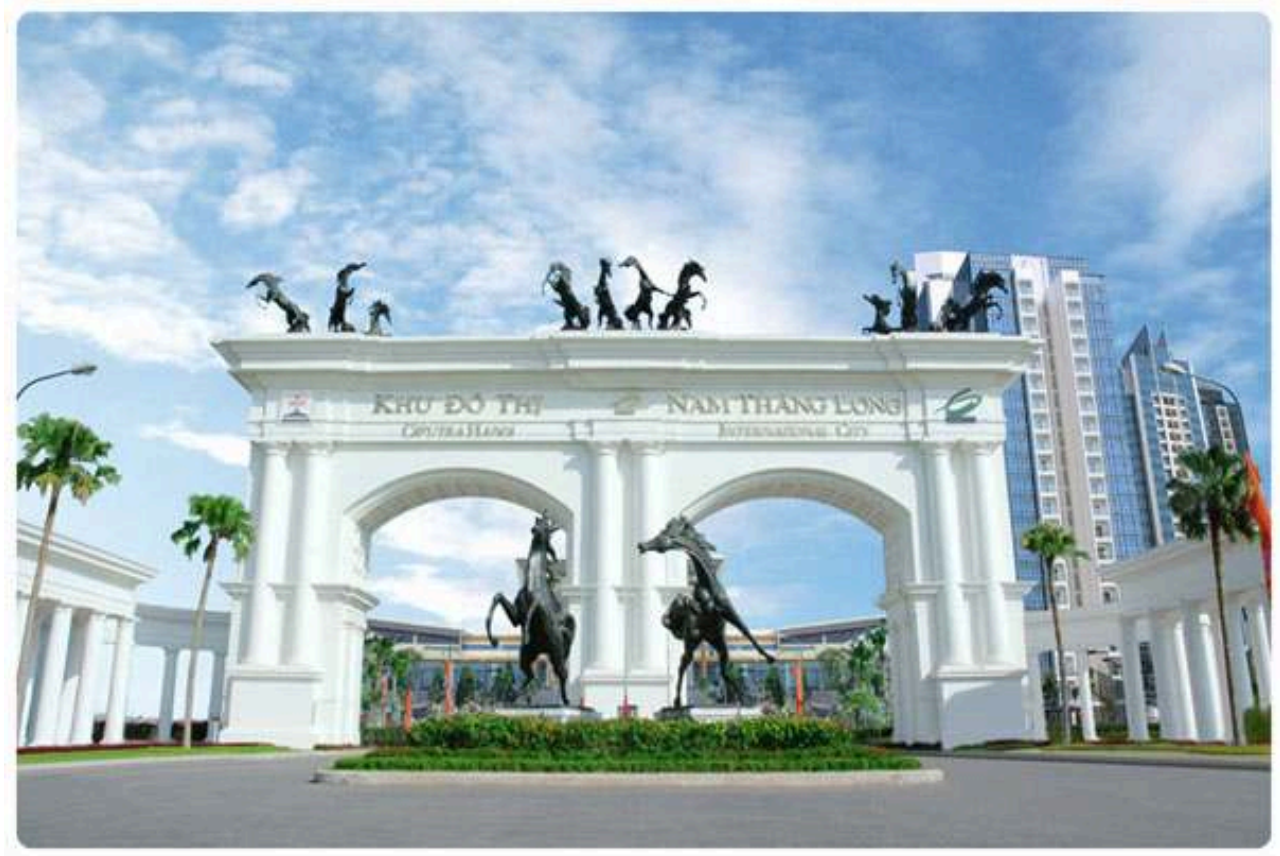

Ciputra Hanoi Urban Area entrance gate (http://ciputrahanoi.info - consulté en janvier 2019)

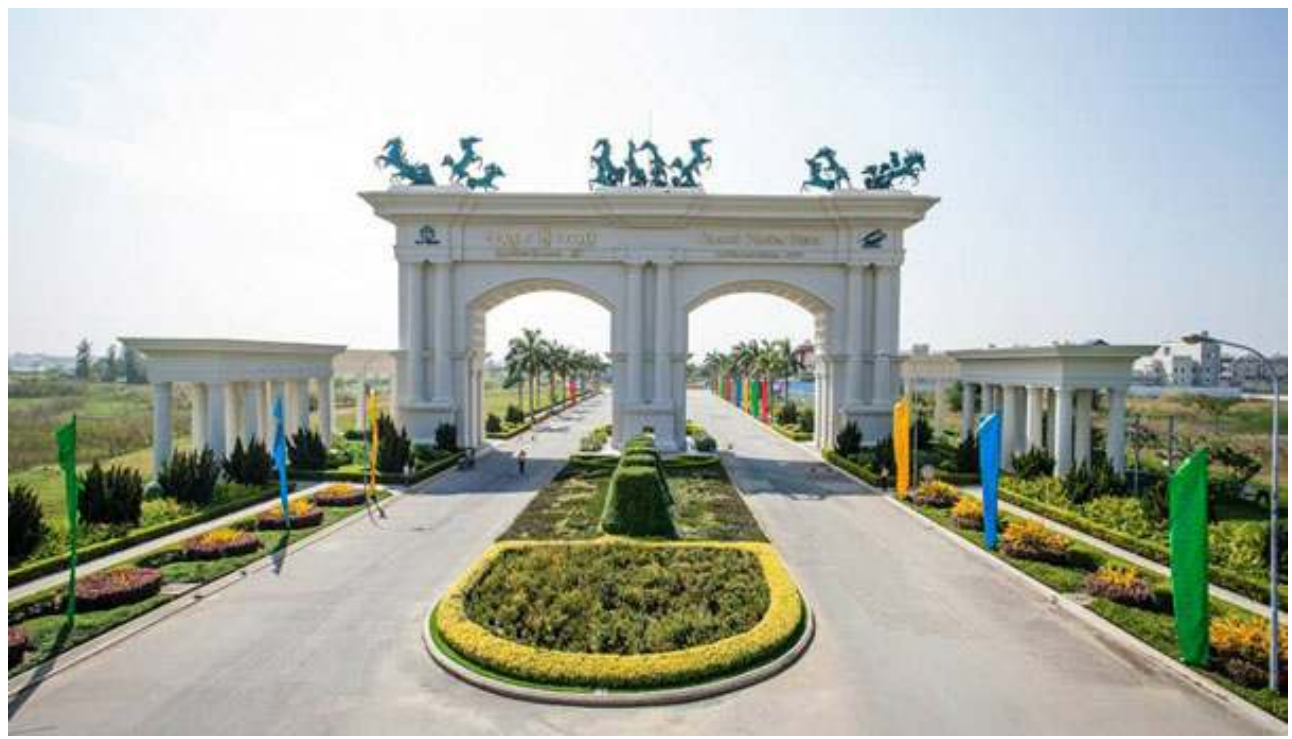

Grand Phnom Penh International City entrance gate (https://grandphnompenh.com/about/ - consulté en janvier 2019)

(sources internet diverses)

Il n'est pas non plus difficile de voir émerger dans les deux pays ce que Lefebvre (ibid.) qualifie de "phallique » en faisant référence au monumental qui vient remplir le vide de sens laissé par le géométrique et le visuel. Le seul exemple (figure 4) des deux condominiums construits sur un modèle identique à Hanoi et Phnom Penh par le groupe indonésien Ciputra confirme à la fois les efforts déployés pour combler ce vide et la proposition d'un sens détaché du lieu, mobilisant une esthétique associée à certaines élites globales. 


\section{B Des usages de l'espace et du droit visibles dans les paysages} problème au sein de la société et le pouvoir semble se satisfaire pleinement de cette distance qu'il laisse se mettre en place et instrumentalise. Au Vietnam, le droit est travaillé de façon bien différente, par le pouvoir qui expérimente et planifie, et par la société qui résiste, l'un et l'autre s'inscrivant dans des «traditions » de pragmatisme d'Etat et de résistances quotidiennes, dont le fonctionnement est attesté depuis la période socialiste (Kerkvliet, 2005). Cet équilibre est aujourd'hui fragilisé par les conflits et par des contre-pouvoirs externes, mais le rôle de l'État reste important et la force publique reste très visible alors qu'elle disparaît devant le marché au Cambodge.

Dans ce pays, les concessions économiques de très grande dimension occupaient près d'un quart du territoire national avant les annulations de 2015 et 2016, repoussant les communautés locales dans des interstices ou les intégrant, parcelle par parcelle, au gré de la politique dite de la "peau de léopard» dont le nom dit bien la conception d'un espace "à la découpe ", pensé pour les investissements plus que pour les résidents. Le long des routes, les murs et barrières déploient leurs linéaires sur des distances bien plus grandes qu'au Vietnam et, dans les villes, on assiste à une surenchère de la fermeture sécuritaire des propriétés (fils barbelés, gardes, miradors, éclairages) qui disent plus la progression encore isolée de la propriété et la reproduction des modèles associés, qu'une réelle insécurité. S'y ajoute un processus très rapide de privatisation des espaces publics qui se traduit par la destruction du patrimoine historique, notamment dans la capitale, ainsi que par la cession de quartiers entiers à des entreprises privées, chargées de les viabiliser et valoriser, comme l'île de Koh Pich, à Phnom Penh, devenue un vaste espace récréatif.

Au Vietnam, l'interférence du privé dans la production d'espaces à vocation publique est indiscutable s'agissant par exemple des innombrables golfs ou des projets immobiliers. Mais l'État maintient un niveau élevé de production d'espaces publics dont la mise en scène donne lieu à une véritable compétition entre collectivités, notamment provinciales (Mellac, 2014). Surgissent ainsi des bâtiments administratifs gigantesques, agrémentés de lacs et de jardins bétonnés, qu'accompagnent des infrastructures, dont des voies de circulation aux dimensions et à l'esthétique encore très soviétiques. La force de l'État se voit encore dans les effets d'une planification qui modifie radicalement la destination des espaces et les spécialise avec une intensité particulièrement élevée, le pays étant par exemple devenu le champion régional, avant même la Chine, du nombre de zones économiques spéciales (Walsh, 2015). Dans un même ordre d'idée, le triplement de l'aire administrative de Hanoï en 2008, devenue alors la plus grande capitale du Sud-Est asiatique, rappelait la force du pouvoir et sa capacité à penser le futur. Depuis quelques années, cette planification est elle-même mise en scène, comme cela existe aussi en Chine, grâce à la réalisation de gigantesques plans en trois dimensions exposés dans le hall de différents offices municipaux ou nationaux ${ }^{9}$.

Cette mise en scène est d'autant plus nécessaire que la politique de distribution égalitaire décidée dans les premiers temps de l'ouverture et menée à son terme sur la quasi-totalité des terres agricoles, produit une micro fragmentation parcellaire qui complique le travail d'expropriation par la multiplication des ayants-droits détenteurs 
de certificats. Cela crée de très nombreux conflits qui entravent les opérations de mise à disposition des terres et créent des antagonismes forts autour de ces opérations avec, d'un côté, les foyers qui comprennent mal que des parcelles allouées leur soient retirées - et soient bien mal compensées - de l'autre des cadres peu habitués aux logiques propriétaires. L'un d'entre eux, membre haut placé de l'administration foncière, indique par exemple: "Ces gens demandent des fortunes de leurs terrains alors qu'ils ont reçu des certificats gratuitement il n'y a pas longtemps. Les autres, les fonctionnaires n'ont toujours que leur salaire! Pourquoi faudrait-il leur donner autant d'argent ?» ${ }^{10}$. Les temps changent et les agents de l'Etat se heurtent, dans leurs projets, à ce que le droit a produit. Activé par les (très) hautes densités rurales, le choix fait par le Vietnam de privilégier d'abord la petite agriculture familiale, produit aussi, un peu partout dans le pays, des paysages caractéristiques.

Figure 5 : Le tracé de la frontière Cambodge-Vietnam dans les hauts plateaux centraux

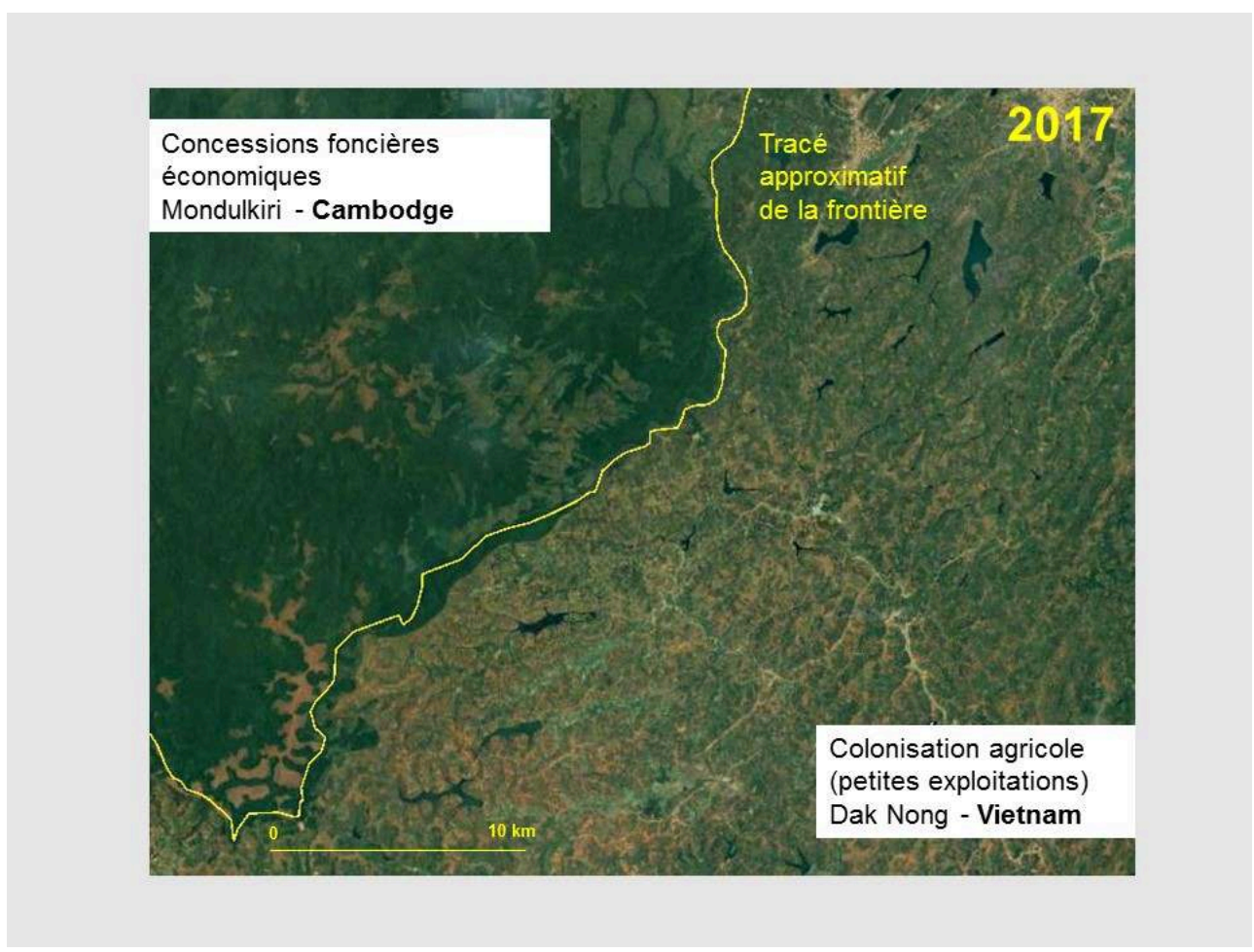

(réalisation M. Mellac d'après une vue Google Earth 2017 - consultée en 2018)

Ainsi, il n'est pas nécessaire, sur une image satellite prise au niveau de la frontière entre le Cambodge et le Vietnam dans la région des hauts plateaux (figure 5), de disposer du tracé de celle-ci pour voir où s'arrête un territoire et où commence l'autre. A l'est, la forêt a été remplacée par une mosaïque dense de petites exploitations et plantations familiales. A l'ouest des pans gigantesques et continus d'une forêt encore dense et cultivée (défriche-brûlis) dont il subsiste encore quelque belles étendues, ont été abattus pour mettre en place des plantations industrielles. 


\section{Conclusion - Les voies multiples vers l'espace mondialisé} dont l'organisation ne reflète plus seulement, ni d'abord, la vie sociale locale. Celle-ci a bien entendu toujours de l'effet, et il aurait fallu quelques pages supplémentaires pour montrer la capacité d'invention des habitants dans le nouvel ordre qui se met en place. Sans nier cette capacité, créatrice de complexité et de différenciations spatiales, le travail accéléré des deux États pour mettre en place des formes d'appropriation foncière compatibles avec le marché, ressort néanmoins comme un élément majeur de transformation de l'espace, celui-ci se dotant progressivement des propriétés de l'espace abstrait. La comparaison du Vietnam et du Cambodge montre néanmoins que les modalités de transition, fortement différenciées, ne sont pas neutres pour l'espace et que le droit joue un rôle dans la façon dont se manifestent spatialement les façons de gouverner le changement. Les transitions se traduisent notamment par une attention plus ou moins grande portée aux modalités de production du droit foncier et à son contenu, largement repris de l'étranger pour le Cambodge ou progressivement inventé pour le Vietnam. Les deux projets d'espace contenus dans les lois sont différents et le sont tant dans la façon dont ils adviennent (progressivement ou non, par copie ou invention) dans le droit que dans les modalités d'appropriation de l'espace sur lesquels ils reposent.

Au Cambodge, où le droit est pourtant réputé ne pas être appliqué, le choix de réduire le régime d'appropriation à la propriété privée a des traductions spatiales nettes, notamment dans la diffusion éparpillée de parcelles privées au sein d'espaces relevant encore dans les faits d'autres régimes d'appropriation, ce qui accentue la violence de la cohabitation. Ce choix est très directement celui des bailleurs. Le Cambodge qui se place sous la tutelle des grandes organisations internationales au début des années 1990, répond en effet rapidement à leurs attentes. Le droit procède alors brutalement et fait advenir la démocratie et la propriété privée par la Constitution. Cela n'est pas étranger à la façon de faire des experts occidentaux qui se pressent dans le pays et proposent des solutions exogènes aux problèmes endogènes. En matière légale cette extériorité est double car renforcée par les façons de faire des juristes dont Latour (2002) notamment, nous dit qu'ils tendent à être tout entiers dans le droit et à ne définir le droit que par lui-même. Ainsi, quels que soient les efforts qu'ils déploient pour comprendre les contextes, les juristes étrangers tendent à produire des textes qui en sont éloignés quand ils ne produisent pas des législations contradictoires (loi de 2001 et code civil) car écrites par des juristes de cultures différentes. La propriété sert aussi les élites qui ont vite compris l'intérêt qu'elles pouvaient avoir à satisfaire aux attentes libérales des bailleurs, la réforme ne constituant en somme pour Hun Sen qu'un changement superficiel à condition que son parti (le CPP) contrôle toujours le réseau administratif descendant jusqu'au village (Slocomb, 2003).

Le Vietnam qui expérimente le socialisme de marché, s'est longtemps tenu à distance des organisations internationales et n'a guère au départ d'autre modèle que la Chine dont le système légal assez similaire (Gillepsie and Chen, 2013). Le Parti qui doit inventer des façons de rendre possible le marché sans mettre à mal sa légitimité, expérimente dans le droit et reste un producteur majeur de l'espace. L'Etat ne s'est pas encore dessaisi de ses capacités de planification et d'allocation des terrains, comme il

Revue Géographique de l'Est, vol. 58 / 1-2 | 2018 
lui est pourtant instamment demandé par les grands bailleurs et les ONG sous couvert notamment de lutte contre la corruption et de lutte pour les droits individuels. Il n'a pas encore non plus totalement distendu le lien entre l'usage des terres et la détention de droits sur elle, préférant à la propriété un régime proche de la possession et par là cher aux penseurs socialistes. Le Vietnam qui accapare les terres de ses voisins (dont le Cambodge) et dont le pouvoir exerce une violence avérée sur la population, n'est certainement pas un exemple à suivre. Mais il montre qu'il existe, pour le développement, des alternatives aux régimes de propriété privée qui font de la terre un bien indifférencié et laissent au marché le pouvoir de production de l'espace. Cela passe jusqu'à présent par une certaine progressivité du droit, avec un changement plus rapide autour de 2000 lorsque le pays donne priorité à l'industrialisation et que la loi (de 2003) ouvre grand l'accès aux terres agricoles et facilité les expropriations. Ce changement a ouvert une période de conflits et a placé les législateurs dans une situation délicate qui s'est manifestée notamment par les longues hésitations ayant entouré la promulgation de la dernière loi foncière en 2013. Pour certains observateurs étrangers, ces hésitations sont interprétées comme le résultat des demandes sociales et des débats qu'ils ont largement participé à animer pour changer le droit dans le cadre du processus de démocratisation qu'expérimente aujourd'hui le pouvoir. Mais les influences exogènes doivent être questionnées et il est important, dans le contexte de migration sans précédent des concepts légaux (Menzel, 2012), de prendre sérieux tant les effets des lois sur l'espace que l'espace qu'elles contiennent. Pour cela

\section{BIBLIOGRAPHIE}

Appadurai A., 2001 [1996], Après le colonialisme, les conséquences culturelles de la globalisation, Paris, Petite Bibliothèque Payot, 333 p.

Blomley N., 2003, « From 'What?' to 'So What?' Law and Geography in Retrospect », in Holder J., Harrison C. (eds), Law and Geography, Oxford, Oxford University Press, Current Legal Issues 2002, vol. 5, p.17-33.

Bourdier F., 2016, "Recent Forms of Social Contestation with Regard to Land Tenure in Cambodia" , Leiden, IIAS, The Newsletter (en ligne), n 73, Spring 2016, p.4-5, consulté le 6 juin 2017, URL : https://iias.asia/sites/default/files/IIAS_NL73_0405.pdf

Butler C., 2009, « Géographie du droit critique et production de l'espace : théorie et méthode selon l'œuvre d'Henri Lefebvre ", in Forest P. (dir), Géographie du droit, épistémologie, développement et perspectives, Québec, Les Presses de l'Université Laval, coll. Dikè, p.137-155.

Carrier A., 2007, Les « lois de la possession » à Phnom Penh : conversion des droits d'usage résidentiel issus du contexte socialiste de réappropriation urbaine (1979-1989) en droits de propriété. Thèse de doctorat en urbanisme, IFU, Université Paris 8, 600 p.

Castellanet C., Diepart J-C., 2015, « The Neoliberal Agricultural Modernization Model », Chiang

Mai, International Academic Conference 5-6 June 2015, Land Grabbing, conflict and agrarian- 
environmental transformations, Conference Paper $\mathrm{n}^{\circ} 55$ (en ligne), consulté le 15 juin 2015, URL : http://www.burmalibrary.org/docs21/CMCP_55-Castellanet_Diepart-red.pdf

Chouquer G., 2011, Terres porteuses. Entre faim de terres et appétit d'espace, Paris, Errances, 250 p.

Dang Hung Vo, Palmkvist, G., 2001, « Sweden-Vietnam Cooperation on Land Administration Reform in Vietnam ", Seoul, New Technology for a New Century International Conference (en ligne), FIG Working Week, 6-11 May 2001, consulté le 13 juin 2008, URL : http://www.fig.net/pub/ proceedings/korea/full-papers/session7/dang-palmkvist.htm

Diepart J-C., Sem, T., forthcoming, The Cambodian Peasantry and the Formalisation of Land Rights: Historical Overview and Current Issues, Paris, CTFD: MAE/AFD, Regards sur le Foncier.

Forest P., 2009, «Introduction », in Forest P. (dir), Géographie du droit, épistémologie, développement et perspectives, Québec, Les Presses de l'Université Laval, coll. Dikè, p.7-20.

Gillepsie J., Chen A., 2013, « Comparing legal development in China and Vietnam: an introduction ", in Gillespie J. and Chen A. (eds.), Legal Reforms in China and Vietnam: A Comparison of Asian Communist Regimes, London: Routledge, p. 1-26.

Hap P., 2010, The Implementation of Cambodia's Laws on Land Tenure, PhD Dissertation, Nagoya University, Graduate School of Law, 200 p.

Hel C., 2008, Les conflits fonciers au Cambodge. Contribution à la connaissance du droit de propriété cambodgien, Thèse pour le doctorat en droit, Université de Paris 1, 417 p.

Hel C., 2012, « Introduction to the Land Law of Cambodia », in Hor K., Menzel J. (eds), Introduction to Cambodian Law, Phnom Penh, Konrad-Adenauer-Stiftung, p.315-335.

Jones G. A., 2003, « Camels, Chameleons, and Coyotes: Problematizing the 'Histories' of Land Law Reform ", in Holder J., Harrison C. (eds), Law and Geography, Oxford, Oxford University Press, Current Legal Issues 2002, vol. 5, p.169-189.

Kerkvliet B.J.T., 2005, The Power of Everyday Politics: How Vietnamese Peasants Transformed National Policy. Ithaca and London, Cornell University Press.

Kiyohara H., 2012, « Civil Code », in Hor K., Menzel, J. (eds), Introduction to Cambodian Law, Phnom Penh, Konrad-Adenauer-Stiftung, p.97-133.

Kong P., 2012, « Overview of the Cambodian Legal and Judicial System and Recent Efforts at Legal and Judicial Reform », in Hor K., Menzel J. (eds), Introduction to Cambodian Law, Phnom Penh, Konrad-Adenauer-Stiftung, p.7-22.

Kork B., 2012, « Cambodian Private Law : a Snapshot », in Hor K., Menzel J. (eds), Introduction to Cambodian Law, Phnom Penh, Konrad-Adenauer-Stiftung, p.73-96.

Latour B., 2002, La fabrique du droit, une ethnographie du Conseil d'Etat, Paris, La découverte, 316 p.

Lefebvre H., 2000 [1974], La production de l'espace, Paris, Anthropos, 485 p.

Le Roy E., 2011, La terre de l'autre, une anthropologie des régimes d'appropriation foncière, Paris, LGDJ Lextenso éditions, MSH, coll. Droit et société, série Anthropologie 54, 441 p.

Melé P., 2009, Pour une géographie du droit en action. Géographie et cultures, n72, 2009, pp. 25-42.

Mellac M., 2014, Vietnam, an Opening Under Control, Lao Cai on the Kunming-Hai Phong Economic Corridor, in Fau N., Sirivanh Khonthapane, Taillard C. (eds), Transnational Dynamics in Southeast Asia: the Greater Mekong Subregion and Malacca Straits Economic Corridors. Singapore: Institute of Southeast Asian Studies, p.143-174. 
Mellac M. et J-C. Diepart, 2017, « Les communs fonciers à l'ère d'internet au Cambodge, une approche par les conflits », Netcom (En ligne), 31-1/2 | 2017, mis en ligne le 01 décembre 2017, consulté le 14 décembre 2017. URL : http://journals.openedition.org/netcom/2657

Mellac M., Fortunel, F., Tran Dac Dan, 2010, La réforme foncière au Viêt Nam, Analyse des jeux d'acteurs et du processus de transformation des institutions aux échelons central et provincial, Paris, AFD/MAE (en ligne), 195p, URL : http://www.foncier-developpement.fr/publication/la-reformefonciere-au-viet-nam-analyse-des-jeux-dacteurs-et-du-processus-de-transformation-desinstitutions-aux-echelons-central-et-provincial-2/

Menzel J., 2012, « Cambodian Law: Some Comparative and International Perspectives ", in Hor K., Menzel J. (eds), Introduction to Cambodian Law, Phnom Penh, Konrad-Adenauer-Stiftung, p.479-496.

Phan Trung Hien, 2014. Must-known Information about Compensation, Subsidies, and Resettlement when State Recovers land, Hanoi, The National Political Publishing House, 324 p.

Pillot D., 1995, « La fin des coopératives : la décollectivisation agricole au Nord Viêtnam ». Les Cahiers d'Outre-Mer, n¹90, avril-juin, p.107-130.

Sassen S., 2014, Expulsions. Brutality and Complexity in the Global Economy, Cambridge-London, The Belknap Press of Harvard University Press, 298 p.

Sekiguchi M., Hatsikano N., 2013. «Land Conflicts and Land Registration in Cambodia », in Unruh J., Williams R. C. (eds), Land and Post-conflict Peacebuilding, London, Earthscan, p.439-450.

Slocomb M., 2003, The People's Republic of Kampuchea 1979-1989, Bangkok, Silkworm Books, 369 p.

Thu L., 2013, « Congress Delays Adoption of Revised Land Law ». VietNamNet Bridge (en ligne), mis en ligne le 24 juin 2013, consulté le 20 novembre 2013, URL : http://english.vietnamnet.vn/ fms/government/77394/congress-delays-adoption-of-revised-land-law.html

Trzcinskil M., Upham, F. K. 2012, The Integration of Conflicting Donor Approaches: Land Law Reform in Cambodia. Journal of International Cooperation Studies, vol.20, $\mathrm{n}^{\circ} 1, \mathrm{p} .129-146$.

Walsh J., 2015, « The Special Economic Zones of the Greater Mekong Subregion », Chiang Mai, International Academic Conference 5-6 June 2015, Land grabbing, Conflict and AgrarianEnvironmental Transformations: Perspectives from East and Southeast Asia, Oral communication.

Wells-Dang A., 2013, Promoting Land Rights in Vietnam: A Multi-Sector Advocacy Coalition Approach. Washington DC, Paper prepared for presentation at the Annual World Bank Conference on Land and Poverty, World Bank, April 8-11, 2013, 25 p.

Wells-Dang A., Pham Quang Tu, Burke A., 2015, « Agrarian Change and Land Tenure in Vietnam through a Political Economy Lens ", Chiang Mai, International Academic Conference 5-6 June 2015, Land grabbing, Conflict and Agrarian-Environmental Transformations: Perspectives from East and Southeast Asia, Conference Paper ${ }^{\circ} 45$ (en ligne), consulté le 6 juin 2015, URL : https://www.iss.nl/ fileadmin/ASSETS/iss/Research_and_projects/Research_networks/LDPI/CMCP_45-WellsDang_et_al.pdf

Williams R.C., 2013, « Title Through Possession or Position? Respect for Housing, Land, and Property Rights in Cambodia ", in Unruh J., Williams R. C. (eds), Land and Post-conflict Peacebuilding, London, Earthscan, p.411-436. 


\section{NOTES}

1. 20 entretiens en vietnamien ont été réalisés au Vietnam entre 2007 et 2015, 25 l'ont été au Cambodge en anglais entre 2014 et 2016. Les textes de loi vietnamiens sont analysés directement en vietnamien et les traductions sont celles de l'auteur. Les textes cambodgiens sont analysés dans leurs versions anglaises et françaises, notamment les traductions en annexe de la thèse de Carrier (2007).

2. En 2014, la superficie des concessions foncières sociales représentait $4 \%$ de celle des ELC pour 12.000 foyers et 8 communautés indigènes avaient reçu un titre collectif. Ce chiffre est monté à 19 en 2017 à comparer aux 166 communautés engagées dans le processus. Entre 2002 et 2012, le titrement a concerné 625.000 foyers seulement pour 317.000 foyers touchés en deux ans par la campagne systématique initiée au titre de l'Ordre 01 quelques mois avant les élections de 2012 et 2013 (Diepart et Sem, forthcoming).

3. Carrier (2007) indique que le texte a été élaboré sous assistance des Nations Unies. Sekiguchi et Hatsukano (2013) estiment plutôt qu'il s'agit d'une production «locale » pilotée, sans aide étrangère, par le gouvernement en place. Une de nos sources d'enquête (Phnom Penh, 02/015) note l'intervention de juristes cambodgiens formés en France et sollicités par le gouvernement.

4. Entretien, Hanoi, 07/2008.

5. Entretien, Hanoi, 07/2008.

6. Deux juristes cambodgiens formés principalement en France indiquent (Entretiens, Phnom Penh, 03 et 04/2015) la participation centrale de juristes français alors que les experts japonais, qu'ils aient été entendus en entretiens (Phnom Penh, 04/2015) ou aient publié à ce sujet (Sekiguchi and Hatsukano, 2013) décrivent un texte produit sous influence anglo-saxonne (ONG et donneurs bilatéraux) dans le cadre d'un projet de l'Asian Development Bank. Trzcinski et Upham (2012) quant à eux indiquent que le texte a été écrit par une équipe financée par la Banque Mondiale.

7. Ce ministère a été créé en 1999 par rassemblement de toutes les compétences foncières, y compris le règlement des conflits fonciers, la justice ayant été jugée incapable de les résoudre.

8. Entretien, Phnom Penh, 03/2015.

9. Pour s'en persuader, voir les différentes expositions organisées à Hanoi : https:// english.vietnamnet.vn/fms/special-reports/11142/-hanoi-master-plan-not-swayed-by-

anyone-.html ou encore http://www.cungquyhoach.vn/trien-lam/trien-lam-mo-hinh-quyhoach-ha-noi-2030-va-tam-nhin-2050.html

10. Entretien, Ho Chi Minh-ville, 12/2014.

\section{RÉSUMÉS}

Cet article s'intéresse à la fabrique des lois foncières considérée comme un moment spécifique de la production de l'espace mondialisé. L'attention portée au domaine foncier se comprend dans le contexte de "mondialisation foncière» dont une manifestation consiste en l'extension, à l'échelon du monde, de la propriété privée foncière. Entrer par le contenu et la production du droit se comprend de son côté par la dimension cognitive du droit et par l'importance des enjeux et des jeux d'acteurs qui se tissent autour de lui. Le travail proposé repose sur la comparaison de la production légale foncière de deux pays voisins et en transition d'Asie du sud-est, le Cambodge et le Vietnam. Par les projets d'espace qu'elles contiennent comme par la façon dont elles ont été 
écrites, les lois foncières nous disent quelque chose des différentes façons dont l'espace peut être mobilisé pour être rendu compatible avec l'économie de marché et le capitalisme.

This paper enlightens the making of land laws as one specific moment of globalised space production. The attention given to land laws is to be understood in the context of the "globalisation of land law" with the extension to the world of private property. The attention given to the texts of law and to their making, is to be understood in relation with the cognitive dimension of the law and with the issues and actors games surrounding law-making. The studied land laws are the ones of two Southeast Asian neighbouring countries, Vietnam and Cambodia. In the two cases, the projects for space encapsulated in the laws and the way laws were produced tell us something of the different way space was mobilized to be made compatible with market economy and capitalism.

Dieser Artikel konzentriert sich auf die Fabrik der Landgesetze und betrachtet sie als eine besondere Zeit der Herstellung des globalisierten Raums. Die Aufmerksamkeit zum Thema Land versteht sich im Zusammenhang der „Land Globalisierung“, welche sich zum Teil durch die weltliche Erweiterung des Landes Privateigentums äußert. Die Analyse des Recht- Inhaltes und Produktion liegt auf die kognitive Kraft des Gesetzes und die Bedeutung der Herausforderungen und Aktionen der Akteure, die sich um ihn herum entwickeln. Die vorliegende Arbeit basiert auf dem Vergleich der Landrecht Produktion von zwei Nachbarn- und Schwellenländer Südostasiens, Kambodscha und Vietnam. Die Landgesetze enthalten spezifische Raumprojekte und wurden in einem bestimmte Art und Weise geschrieben. Dadurch erzählen sie uns etwas über die verschiedenen Möglichkeiten, in denen Raum mobilisiert werden kann, um mit der Marktwirtschaft und Kapitalismus kompatibel gemacht zu werden.

\section{INDEX}

Mots-clés : droit, foncier, espace, mondialisation, Vietnam, Cambodge

Schlüsselwörter : Recht, Land, Raum, Globalisierung, Vietnam, Kambodscha

Keywords : Law, Land, Space, Globalisation, Vietnam, Cambodia

\section{AUTEUR}

\section{MARIE MELLAC}

Passages - UMR 5319 (CNRS - Université Bordeaux Montaigne) Maison des Suds12, esplanade des AntillesDomaine universitaire33607 - Pessac-cédex 